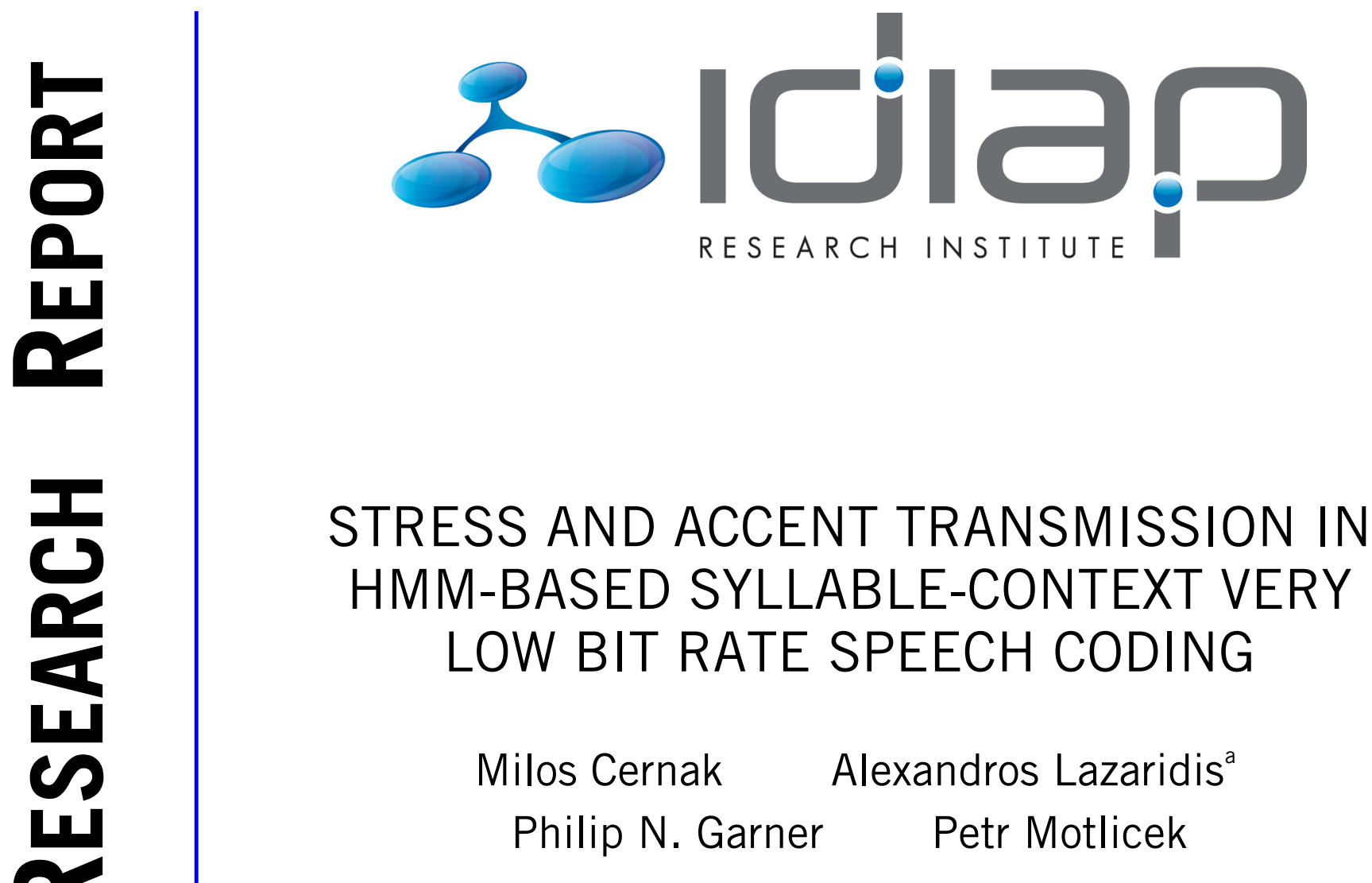

Idiap-RR-10-2014

JUNE 2014

adiap Research Institute 



\title{
Stress and Accent Transmission In HMM-Based Syllable-Context Very Low Bit Rate Speech Coding
}

\author{
Milos Cernak, Alexandros Lazaridis, Philip N. Garner, Petr Motlicek
}

June 23, 2014

\begin{abstract}
In this paper, we propose a solution to reconstruct stress and accent contextual factors at the receiver of a very low bit-rate speech codec built on recognition/synthesis architecture. In speech synthesis, accent and stress symbols are predicted from the text, which is not available at the receiver side of the speech codec. Therefore, speech signal-based symbols, generated as syllable-level log average F0 and energy acoustic measures, quantized using a scalar quantization, are used instead of accentual and stress symbols for HMM-based speech synthesis. Results from incremental real-time speech synthesis confirmed, that a combination of F0 and energy signal-based symbols can replace their counterparts of text-based binary accent and stress symbols developed for text-to-speech systems. The estimated transmission bit-rate overhead is about 14 bits/second per acoustic measure.
\end{abstract}

\section{Introduction}

Very low bit-rate (VLBR) speech coders operate at bit-rates $\leqslant 600$ bits per second (b/s). Considering the Shannon'source coding theorem, the minimal bit-rate is based on the entropy of the phonemes and basic prosodic features, together on the order of $100 \mathrm{~b} / \mathrm{s}$ uncompressed; this is an "operating point" of our work. The prototype of our VLBR coder is real-time and produces intelligible speech with low communication delay ${ }^{1}$.

For achieving this very low bit-rate, parameteric speech coding has to be used. VLBR speech coders with speech recognition and synthesis architectures belong to the most popular approaches. In our work we use phoneme recognition as a transmitter, and phoneme synthesis as a receiver. Transmission of pitch and duration of the symbols is required to recover the original prosody. While corpus-based techniques have been applied in the past for VLBR speech coding systems (e.g., Baudoin and El Chami (2003)), HMM-based speech synthesis systems (HTS) are beneficial from an adaptation point of view, and the system footprint Tokuda et al. (1998).

In our recent work on VLBR speech coding Cernak et al. (2013a,b), we expressed the aim to unify the transmitted information on the syllable context level. We hypothesised that unifying context across all levels of transmitted information may decrease the overall bit rate of the speech coder, while allowing acceptable communication delay. In this paper we focus on one module of the receiver, and specifically on the context reconstruction from the transmitted parameters. The context has significant impact on the naturalness of the speech generated by an integrated HTS system. We propose a solution to reconstruct stress and accent contextual factors at the receiver side. Both factors are normally predicted by a text analyzer (e.g., using a word dictionary for stress and a prediction model for accent). The text in phoneme based recognition and synthesis is not available, however, the speech signal is. Therefore our main research hypothesis is that the acoustic correlates of text-based accent and stress prediction (we call them speech signal-based) in HTS synthesis can perform as well as text-based contextual factors. For validating the hypothesis, we use the quantized average log pitch (F0) and energy labels in the syllable context HTS modelling.

The idea of using speech signal-based labels has recently been applied to low bit rate F0 coding Nose and Kobayashi (2011). More closely to our work, F0 quantized symbols were used for unsupervised labelling of accentual context in Nose et al. (2010). The F0 sequence for synthesis from text was generated using an average voice model. We extended the idea of quantized FO by using multiple signal-based symbols (F0 and energy), and passing from phone-level to syllable-level quantization that fits our very

\footnotetext{
${ }^{1}$ Some examples available at https://www.idiap.ch/project/recod/demo/incremental-coding/
} 
low bit rate speech coding framework. The novel aspect of our work is a combination of different signalbased measures that aim to replace the conventional text-based contextual factors that are not available at the receiver side of the codec, such as factors related to word and phrase contextual factors. While the effectiveness of quantized F0 symbols was proved in Nose et al. (2010) for a syllable-timed language, we found that using a combination of more acoustic measures is necessary for stress-timed languages (see description in the next section). We evaluated the accent and stress modelling for English language.

The paper is structured in the following way. The next Sec. 2 introduces the stress and accent contextual factors and their acoustic signal-based correlates. The Sec. 3 presents an analysis of predictability power of acoustic correlates based on the normalised mutual information measure. Sec. 4 describes the experiments and results, and finally Sec. 5 concludes the paper with the discussion and future work outline.

\section{Stress and accent contextual factors}

In this work, by the term stress, we refer to the lexical stress of a word, which is the stress placed on syllables within words. In some languages, such as English, a word might have a secondary stress, distinct from the primary one. Languages can be classified into two main categories, syllable-timed and stress-timed languages, depending on whether the duration of every syllable is equal or the duration of the intervals between two stressed syllables are equal, respectively Pike (1945); Abercrombie (1967). In early work, Fry Fry $(1955,1958)$ showed that stress was highly correlated to acoustic variations in speech, such as increased duration and higher intensity and moreover higher pitch values. Gordon Matt (2014), showed that in stress-timed languages, such as English, stresses assigned at the word level are correlated to speech properties such as an increased duration of the stressed phonemes or syllables, higher intensity values, and/or hyper-articulation, rather than with pitch variations on these syllables. Additionally, Sluijter and van Heuven Agaath and Vincent (1996) concluded that, in English, pitch is correlated to phrase-level pitch accent rather than to word-level stress. The controversy between these two above-mentioned views is caused by the fact that Fry's research was conducted on words positioned on parts of phrases that conveyed not only word-level stress but also phrase-level prominence, leading to the conclusion that stress was also correlated to higher pitch values.

On the other hand, accent refers to the phrase- or sentence-level prominence given to a syllable. The syllables conveying phrasal prominence are often called pitch accented syllables. In the rest of the paper, with the term accent we refer to the pitch accent, which is the phrasal prominence distinct from tones correlated to the boundaries of intonational phrases. Pitch accents mainly convey semantic information such as focus and emphasis. In some cases, a stressed syllable can be promoted to pitch accented syllable based just on it's position in a phrase or on the focus/emphasis the speaker intends to give to the specific part of the sentence to convey a specific message Matt (2014).

In our work, we used syllable-based F0 and energy acoustic measures as acoustic correlates of the accent and stress features. Although the duration was also reported as an important measure, we omitted duration from this work. The duration of syllable is correlated to the number of phonemes in a syllable, a contextual factor already used in the HTS training.

The accent and stress HTS contextual features could take binary values that represent accented and/or stressed syllable. We generated F0 $p_{i}$ and energy $e_{i}$ labels by averaging their values per syllable, and quantizing the average values using a scalar quantization (see Sec. 4.2). The quantization code-book, created per speaker, was linearly spaced between the $\mu-3 \sigma$ and $\mu+3 \sigma$ boundaries, where $\mu$ is the mean and $\sigma$ is the standard deviation of all measurements belonging to the training data of a particular speaker. So the quantization book was created per acoustic measure and speaker. To simplify the creation of the question set used for context clustering of these signal-based labels, 3-bit quantization was used resulting in 8 different labels for each acoustic measure.

\section{Analysis of information related to signal-based labels}

This section describes the analysis of signal-based labels, based on Mutual Information (MI) of the textbased and signal-based labels. The work in Nose et al. (2010) showed that quantized F0 are effective for accent prediction for a syllable-timed language. The main goal of this section is to estimate the predictability power of individual and combined quantized F0 and energy contextual factors for English, a stress-timed language. 
Conventional stress and accent contextual factors are predicted from the text to be synthesised, and the binary labels $c \in 0,1$ are assigned to the current and previous syllable in a phrase. The proposed average quantisized F0 $p_{i}$ and energy $e_{i}$ features are calculated from the speech signal measures, and the labels $M_{i} \in\{0,1, \ldots, 7\}$ were assigned also to the current and previous syllable (8 labels resulted from use of 3-bit code-books). To combine the $p_{i}$ and $e_{i}$ features (to capture, e.g., higher pitch and lower energy) into a single label, we constrained $M_{i}=p_{i}=e_{i}$, where the value of the label in question was the same for both acoustic measures. It simplified the construction of the question set for the context clustering as well.

The MI as a measure of the conventional stress and accent labels $C$ and speech signal measure-based $M_{i}$ labels can be defined as:

$$
\mathrm{I}\left(\mathrm{C} ; \mathrm{M}_{\mathrm{i}}\right)=\sum_{\mathrm{c}} \sum_{m \in M_{i}} p(\mathrm{c}, \mathrm{m}) \log _{2}\left(\frac{\mathrm{p}(\mathrm{c}, \mathrm{m})}{\mathrm{p}(\mathrm{c}) \mathrm{p}(\mathrm{m})}\right),
$$

where $p(c, m)$ is a joint probability of $C$ and $M_{i}$, and $p(c)$ and $p(m)$ are their marginal probabilities. The MI is a measure of information in bits that conveys $M_{i}$ about $C$, and it is normalised by the mutual information measure with the entropy of $\mathrm{C}$ defined as:

$$
H(C)=-\sum_{c} p(c) \log _{2} p(c) .
$$

We evaluated the normalised measure $\frac{\mathrm{I}\left(\mathrm{C} ; \mathrm{M}_{i}\right)}{\mathrm{H}(\mathrm{C})}$ for the following classes (options) of C:

1. $C_{a}$, where $C_{a}=a c c e n t$, and is a measure of information in bits that conveys $M_{i}$ about conventional accent labels,

2. $C_{s}$, where $C_{s}=$ stress, and is a measure of information in bits that conveys $M_{i}$ about conventional stress labels,

3. $\mathrm{C}_{\mathrm{s} \wedge \mathrm{a}}$, where $\mathrm{C}_{\mathrm{s} \wedge \mathrm{a}}=$ stress $\wedge$ accent, and is a measure of information in bits that conveys $M_{i}$ about the intersection of conventional stress and accent labels, i.e., $C_{a} \cap C_{s}=\left\{c: c \in C_{a} \wedge c \in C_{s}\right\}$.

4. $C_{s \mid a}$, where $C_{s \mid a}=$ stress|accent, and is a measure of information in bits that conveys $M_{i}$ about the union of conventional stress and accent labels, i.e., $\mathrm{C}_{\mathrm{a}} \cup \mathrm{C}_{\mathrm{s}}=\left\{\mathrm{c}: \mathrm{c} \in \mathrm{C}_{\mathrm{a}}\right.$ or $\left.\mathrm{c} \in \mathrm{C}_{\mathrm{s}}\right\}$,

Table 1 shows the normalised MI values of the male bdl testing speaker from the CMU-ARCTIC speech database Kominek and Black (2004).

Table 1: Normalised MI values of bdl voice.

\begin{tabular}{|l|c|c|c|}
\hline \multirow{2}{*}{$\frac{\mathrm{I}\left(\mathrm{C} ; \mathrm{M}_{i}\right)}{\mathrm{H}(\mathrm{C})}$} & \multicolumn{3}{|c|}{$\mathrm{bdl}$} \\
\cline { 2 - 4 } & $p_{i}$ & $e_{i}$ & $p_{i}, e_{i}$ \\
\hline \hline $\mathrm{C}=\mathrm{C}_{\mathrm{a}}$ & 10.1 & 8.2 & 16.7 \\
\hline $\mathrm{C}=\mathrm{C}_{\mathrm{s}}$ & 10.9 & 7.4 & 18.3 \\
\hline $\mathrm{C}=\mathrm{C}_{\mathrm{a} \wedge \mathrm{s}}$ & 11.4 & 9.6 & 17.9 \\
\hline $\mathrm{C}=\mathrm{C}_{\mathrm{a} \mid \mathrm{s}}$ & 12.4 & 8.5 & $\mathbf{2 0 . 6}$ \\
\hline
\end{tabular}

From the normalised MI values we see that (a) $p_{i}$ is more informative than $e_{i}$ and (b) individual $p_{i}$ and $e_{i}$ values are less predictive than their combination. The best predicted syllables using the signalbased labels are those which are either accented or stressed, i.e., those represented by conventional stress or accent label class $C_{a \mid s}$. From this analysis we can conclude that proposed $M_{i}$ labels have enough predictability power to replace the conventional text-based stress and accent labels. In that way we do not model explicitly stress and accent. Rather we hypothesise, that the context clustering does the job in a data-driven manner, and selected contextual questions about $M_{i}$ values will reflect the information about actual conventional stress and accent features.

\section{Experimental setup}

The experimental setup we used is depicted in Fig. 1. Focusing on evaluation of the contextual factors of the HTS modelling that is at the receiver side, we abstracted the transmitter side and used the true input 


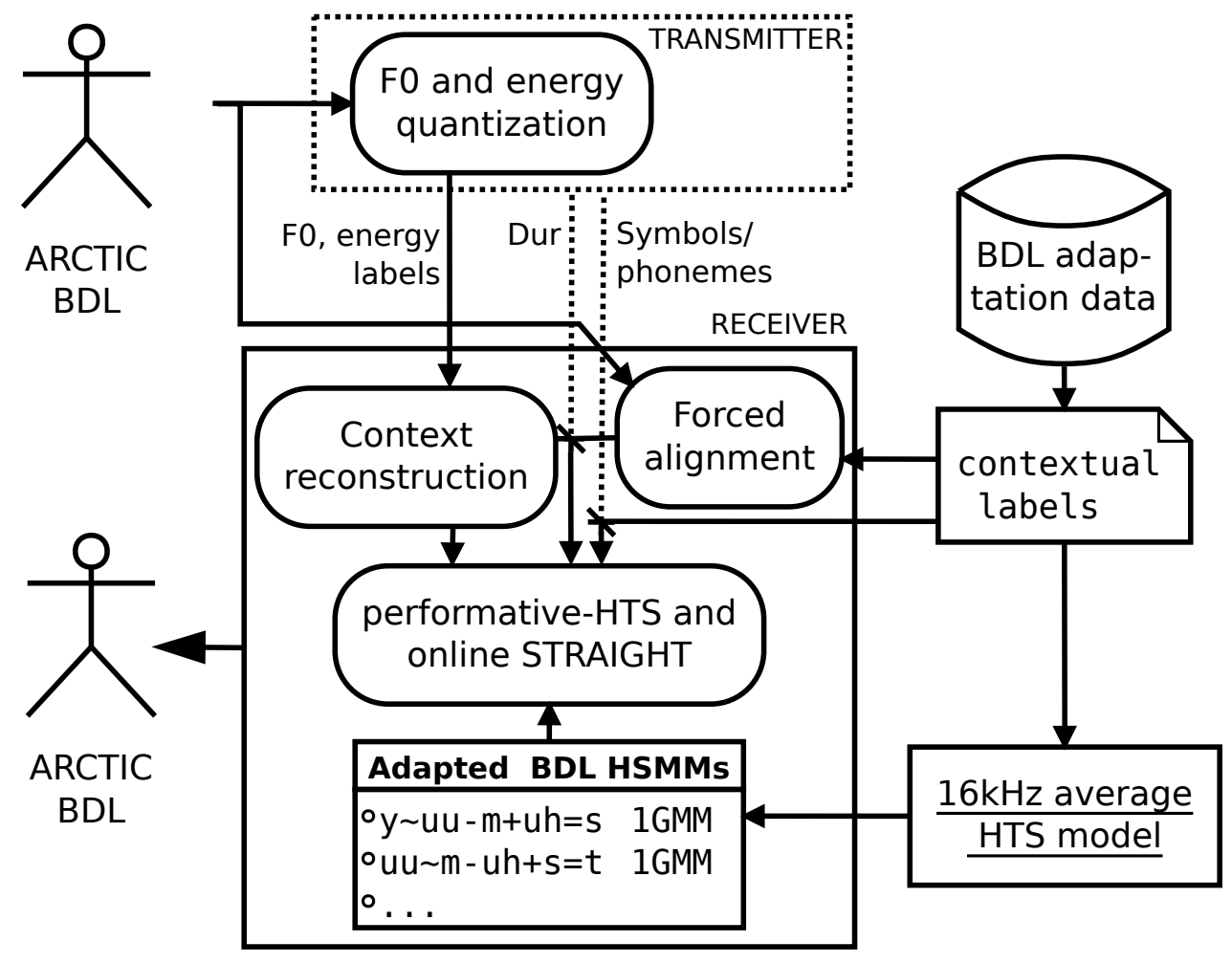

Figure 1: VLBR speech coding experimental setup with recognition-synthesis architecture, abstracting the transmitter (dotted lines) except for FO and energy quantization.

to the receiver, i.e., phoneme sequence from the manually prepared syllable context labels, duration of phonemes from the forced alignment against natural speech, and original pitch values.

As our objective was to build an incremental real-time speech coder, we used the incremental p-HTS system speech parameter generation with 2 previous label smoothing Astrinaki et al. (2012). Speech samples were finally re-synthesized using a real-time incremental STRAIGHT re-synthesis Kawahara et al. (2001). In this way speech samples are generated frame by frame with each processed phoneme.

\subsection{Contextual factors}

Our recent work on contextual factors in HTS concluded that the context above syllable (the word and phrase context) is less important Cernak et al. (2013a). To evaluate the proposed syllable-context signalbased labels, we have designed 3 kinds of synthesis systems integrated within the receiver:

1. Baseline: No accent/stress context, the system used only the full phonetic context,

2. Conventional: Accent/stress context, the system extended the baseline system with the conventional accent and stress labels,

3. Proposed: signal-based label context, the system extended the baseline system with the proposed signal-based labels.

Tab. 2 lists contextual factors for all the three systems.

The combination of signal-based qunatized F0 $p_{i}$ and quantized energy $e_{i}$ factors for context clustering was achieved by listing all possible values of $p_{i}$ and $e_{i}$ within one question, with constrain $M_{i}=p_{i}=e_{i}$ (as described in Sec. 3 ).

\subsection{Generation of signal-based labels}

For training and testing of the baseline and conventional systems, the contextual labels provided by the CMU-ARCTIC database were used. For the proposed system, new labels were generated as follows.

First, the code-books for average log F0 and log energy were created. All log F0 measurements of the training set were extracted using the TEMPO method of Kawahara et al. (1999) using $5 \mathrm{~ms}$ frame 
Table 2: Description of contextual factors used in the proposed syllable-context synthesis systems. The abbreviations PS and CS stand for the previous and current syllable, respectively.

\begin{tabular}{|l|l|l|}
\hline Baseline & Conventional & Proposed \\
\hline \hline - full phoneme context & - full phoneme context & - full phoneme context \\
- the number of phonemes in the PS & - the number of phonemes in the PS & - the number of phonemes in the PS \\
- the number of phonemes in the CS & - the number of phonemes in the CS & - the number of phonemes in the CS \\
- vowel name in the CS & - vowel name in the CS & - vowel name in the CS \\
& - whether the PS stressed $\{0,1\}$ & - quantized F0 $p_{i}$ label of the PS $\{0, \ldots, 7\}$ \\
& - whether the PS accented $\{0,1\}$ & - quantized energy $e_{i}$ label of the PS $\{0, \ldots, 7\}$ \\
& - whether the CS stressed $\{0,1\}$ & - quantized F0 $p_{i}$ label of the CS $\{0, \ldots, 7\}$ \\
& - whether the CS accented $\{0,1\}$ & - quantized energy $e_{i}$ label of the CS $\{0, \ldots, 7\}$ \\
\hline
\end{tabular}

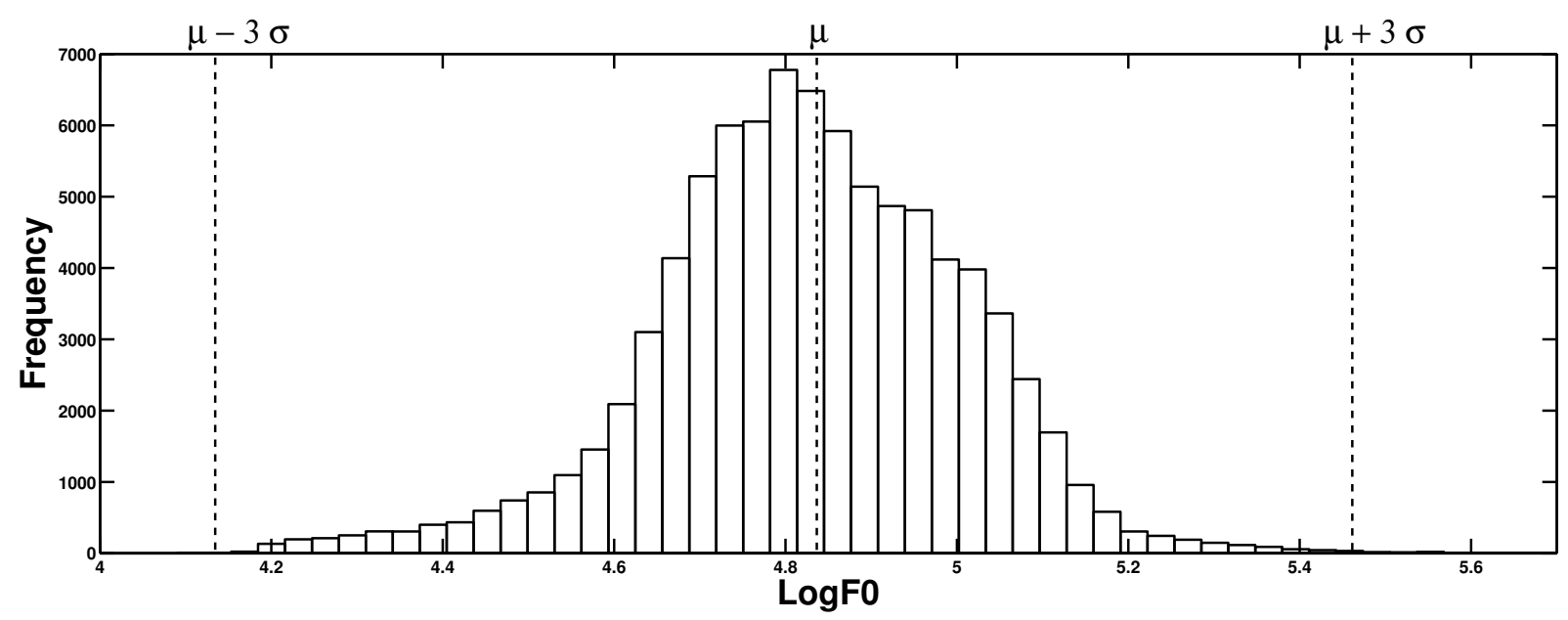

Figure 2: A histogram of the syllable-average log FO values for the code-book creation.

shift. The syllable boundaries were extracted from the contextual labels provided by the CMU-ARCTIC database, and average values were calculated per syllable. Fig. 2 shows the histogram of calculated values together with the boundaries for the 3-bit scalar quantization $(\mu-3 \sigma, \mu+3 \sigma)$ for the bdl speaker. Similarly, the syllable-average log energy values were calculated using the signal-processing tracter tool Garner and Dines (2010) and the log energy code-book was created.

In the next step, all F0 and energy measurements were quantized and new signal-based labels were created for both training and testing sets. We replaced the conventional stress and accent labels with these new labels (see Tab. 2), and used them for training and testing of the proposed system.

\subsection{HTS training}

For building the HMM models, the HTS v. 2.1 toolkit HTS (2010) was used. Specifically, the implementation from the EMIME project Wester et al. (2010) was taken. The speech data which were used had $16 \mathrm{kHz}$ sampling frequency. Five-state, left-to-right, no-skip HSMMs were used. The speech parameters which were used for training the HSMMs were 39 order cepstral coefficients, log-F0 and 21-band aperiodicities, along with their delta and delta-delta features, extracted every $5 \mathrm{~ms}$.

For the experiments, the CMU-ARCTIC database was used. For each of the three systems presented in this work (baseline, conventional and proposed) an American English average model was built using five speakers, including 3 males (bdl, jmk and rms) and 2 females (clb and slt). The unilex Fitt (2000) phone-set was used, consisting of 41 phones. Each utterance with unique id was assign to:

- the training set, if $0 \leqslant i d \leqslant 450$,

- the test set, if $450<i d \leqslant 500$, and

- the adaptation set, if $i d>500$.

In this way, the training set of the average model contained 4493 sentences (some corrupted utterances were excluded from the training). The bdl speaker was selected as a testing speaker. The bdl adaptation 


\section{$\square$ Baseline $\square \mathrm{EQ} \square$ Conventional}

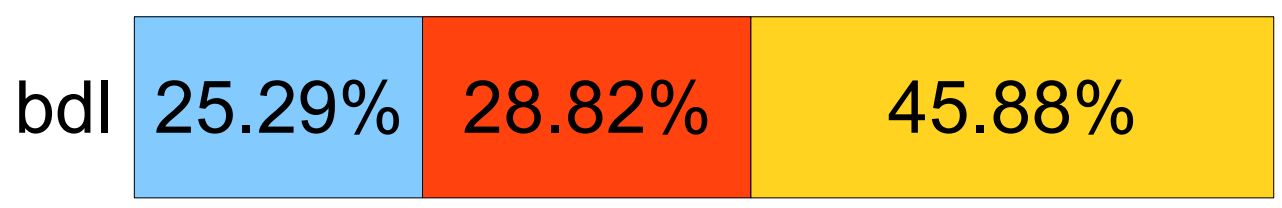

Figure 3: ABX subjective evaluation test results (in percentages) for the comparison between the baseline and conventional systems, for bdl speaker.

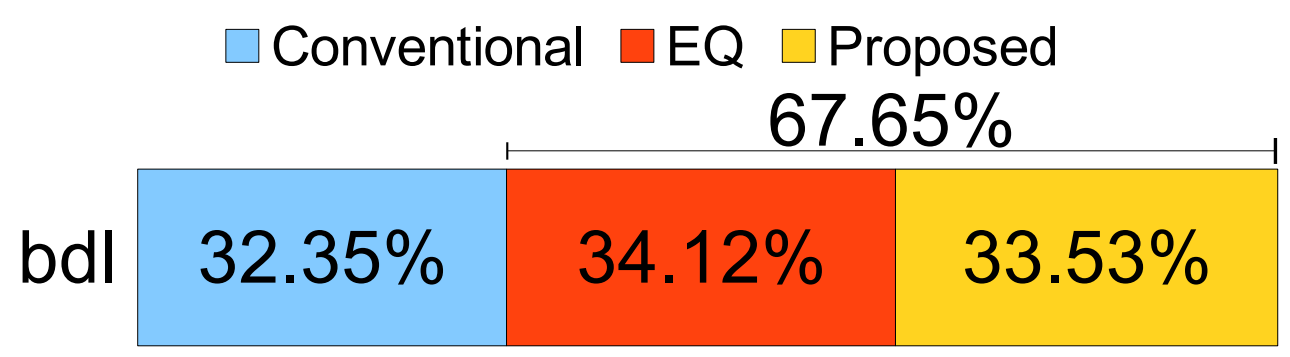

Figure 4: ABX subjective evaluation test results (in percentages) for the comparison between the conventional and proposed systems, for bdl speaker.

set of 131 sentences was used for adapting each of the three average models, resulting into three models used for the three different systems described above. Finally the bdl test set of 100 sentences was used for evaluating the three systems.

\subsection{Evaluation and results}

For the subjective evaluation of the three systems, two ABX tests were conducted. According to Grancharov and Kleijn (2008), the ABX test is suitable for rating small degradation using a continuous impairment scale, and expert (trained) listeners should be used. In our case we were interested if there is some small degradation of the proposed system, comparing to the conventional system.

In the first $\mathrm{ABX}$ test, listeners were asked to choose between speech samples coming from two systems, the baseline and the conventional systems. The motivation behind the first test was to investigate whether the use of stress and accent information based on the textual labels will improve the quality of the synthesized speech. In the second $\mathrm{ABX}$ test, listeners were asked to choose between speech samples produced from the conventional and the proposed systems. The motivation for the second ABX test was to see whether the use of stress and accent information based on the speech signal on the encoder's part rather than based on textual labels, will effect the overall quality of the synthetic speech on the decoder's side.

In both tests 17 listeners, most of them from Idiap speech group, participated. In each test, the listeners were asked to listen for each pair of sentences the two samples (as many times as they wanted), and choose between the two samples in terms of the overall quality. Additionally, the listeners could choose a third option, "both samples sound the same", if they had no preference between them. For both tests the same 10 sentences from the test set were used ${ }^{2}$, concluding to 30 samples in total, i.e., 10 samples for each of the three systems, baseline, conventional and proposal respectively.

Fig. 3 presents the results of the first test. The conventional system significantly outperforms the baseline system. These results clearly show that, as it was expected, the use of stress and accent information based on the textual labels, i.e., the conventional labels, improve the quality of the synthesized speech.

Fig. 4 presents the results of the second listening test. The results obtained indicate that the performances are not significantly different, i.e., the proposed system with labels inferred from speech performed similarly as the conventional system developed to text-to-speech synthesis with labels inferred from text. As stated above, the motivation for the second ABX test was to investigate if the use of stress and accent information based on the speech signal on the transmitter's part will deteriorate the overall

\footnotetext{
${ }^{2} \mathrm{~A}$ subset of voice samples is available at http://www.idiap.ch/project/recod/demo/stress-encoding.
} 
quality of the synthetic speech on the decoder's side in respect to the system using stress and accent as conventional text-based contextual factors. Under this hypothesis, the comparison we are focused on in the second $A B X$ test is between the preference score of the conventional system and the combination of the scores of the EQ case and the proposed system (i.e., we classify EQ case as success). From this point of view, the proposed system outperforms the conventional one in terms of preference score, with $67.65 \%$ over $32.35 \%$, respectively. These results clearly validate our hypothesis that speech signal-based stress and accent features could perform as well as text-based contextual factors.

The bdl test set contained 1312 syllables in 274 seconds of speech. In average, there were so 4.8 of syllables per second. Using the 3-bit quantization book, each acoustic measure needed around $14 \mathrm{~b} / \mathrm{s}$ that we may consider as the transmission overhead per acoustic measure.

\section{Conclusions}

The results clearly validate our hypothesis that speech signal-based stress and accent features could perform as well as text-based contextual factors. Moreover, listeners more prefered the proposed system with quantized syllable-level log average F0 and energy acoustic measures, than conventional stress and accent features as provided by the TTS front-end. The proposed solution works well for speech coding and is probably less effective for speech synthesis from text.

We believe that adding new syllable-based acoustic measures, such as voice quality measures, that might correlate with some other prosodic or emotional features can further improve the naturalness of the encoded speech.

\section{Acknowledgements}

This research was supported by the RECOD project by armasuisse, the Procurement and Technology Center of the Swiss Federal Department of Defence, Civil Protection and Sport and under the SIWIS project by the Swiss National Science Foundation.

\section{References}

HMM-based speech synthesis system version 2.1. 2010. URL http://hts.sp.nitech.ac.jp.

David Abercrombie. Elements of general phonetics. Edinburgh: Edinburgh University Press, 1967.

M.C. Sluijter Agaath and J. van Heuven. Vincent. Spectral balance as an acoustic correlate of linguistic stress. Journal of the Acoustical Society of America, 100:2471-2485, 1996.

M. Astrinaki, N. d'Alessandro, B. Picart, T. Drugman, and T. Dutoit. Reactive and continuous control of HMM-based speech synthesis. In Spoken Language Technology Workshop (SLT), 2012 IEEE, pages 252-257. IEEE, December 2012. ISBN 978-1-4673-5125-6. doi: 10.1109/slt.2012.6424231. URL http://dx.doi.org/10.1109/slt.2012.6424231.

Geneviève V. Baudoin and F. El Chami. Corpus based very low bit rate speech coding. In Proc. of ICASSP, volume 1, pages I-792-I-795 vol.1. IEEE, April 2003. ISBN 0-7803-7663-3. doi: 10.1109/icassp.2003. 1198900. URL http://dx.doi.org/10.1109/icassp.2003.1198900.

M. Cernak, P. Motlicek, and P. N. Garner. On the (UN)importance of the contextual factors in HMMbased speech synthesis and coding. In Proc. of ICASSP, pages 8140-8143. IEEE, May 2013a. doi: 10.1109/icassp.2013.6639251. URL http://dx.doi.org/10.1109/icassp. 2013.6639251.

Milos Cernak, Xingyu Na, and Philip N. Garner. Syllable-Based Pitch Encoding for Low Bit Rate Speech Coding with Recognition/Synthesis Architecture. In Proc. of Interspeech, pages 3449-3452, August 2013b. URL http://www.isca-speech.org/archive/interspeech_2013/i13_3449.html.

S. Fitt. Documentation and user guide to unisyn lexicon and post-lexical rules. Technical report, Center for Speech Technology Research, University of Edinburgh, Tech. Rep., 2000.

D.B. Fry. Duration and intensity as physical correlates of linguistic stress. Journal of the Acoustical Society of America, 27:765-768, 1955. 
D.B. Fry. Experiments in the perception of stress. Language and Speech, 1:120-152, 1958.

P. N. Garner and J. Dines. Tracter: a lightweight dataflow framework. In Proc. of Interspeech, pages 1894-1897, Makuhari, Japan, 2010.

Volodya Grancharov and W. . Bastiaan Kleijn. Speech Quality Assessment. In Jacob Benesty, Sondhi, and YitengArden Huang, editors, Springer Handbook of Speech Processing, pages 83-100. Springer Berlin Heidelberg, 2008. doi: 10.1007/978-3-540-49127-9\_5. URL http://dx.doi.org/10.1007/ 978-3-540-49127-9_5.

H. Kawahara, H. Katayose, A. de Cheveigné, and R. D. Patterson. Fixed point analysis of frequency to instantaneous frequency mapping for accurate estimation of F0 and periodicity. In Proc. of Eurospeech, Budapest, Hungary, 1999.

H. Kawahara, Jo Estill, and O. Fujimura. Aperiodicity extraction and control using mixed mode excitation and group delay manipulation for a high quality speech analysis, modification and synthesis system straight. In MAVEBA, 2001.

J. Kominek and A. Black. The CMU Arctic speech databases. In Proc. of 5th ISCA Speech Synthesis Workshop, pages 223 - 224, 2004.

Gordon Matt. Disentangling stress and pitch accent: Toward a typology of prominence at different prosodic levels. in Harry van der Hulst (ed.). To appear, In Word Stress: Theoretical and Typological Issues, Oxford University Press, 2014.

T. Nose and T. Kobayashi. Very low bit-rate F0 coding for phonetic vocoder using MSD-HMM with quantized F0 context. In Acoustics, Speech and Signal Processing (ICASSP), 2011 IEEE International Conference on, pages 5236-5239. IEEE, May 2011. ISBN 978-1-4577-0538-0. doi: 10.1109/icassp. 2011.5947538. URL http://dx.doi.org/10.1109/icassp. 2011.5947538.

T. Nose, K. Ooki, and T. Kobayashi. HMM-based speech synthesis with unsupervised labeling of accentual context based on F0 quantization and average voice model. In Acoustics Speech and Signal Processing (ICASSP), 2010 IEEE International Conference on, pages 4622-4625. IEEE, March 2010. ISBN 978-14244-4295-9. doi: 10.1109/icassp.2010.5495548. URL http://dx.doi.org/10.1109/icassp. 2010. 5495548.

Kenneth L. Pike. The intonation of American English. University of Michigan Publications in Linguistics 1. Ann Arbor: University of Michigan Press, 1945.

K. Tokuda, T. Masuko, J. Hiroi, T. Kobayashi, and T. Kitamura. A very low bit rate speech coder using HMM-based speech recognition/synthesis techniques. In Proc. of ICASSP, volume 2, pages 609-612 vol.2. IEEE, May 1998. ISBN 0-7803-4428-6. doi: 10.1109/icassp.1998.675338. URL http://dx. doi.org/10.1109/icassp.1998.675338.

Mirjam Wester, John Dines, Matthew Gibson, Hui Liang, Yi-Jian Wu, Lakshmi Saheer, Simon King, Keiichiro Oura, Philip N. Garner, William Byrne, Yong Guan, Teemu Hirsimäki, Reima Karhila, Mikko Kurimo, Matt Shannon, Sayaka Shiota, Jilei Tian, Keiichi Tokuda, and Junichi Yamagishi. Speaker adaptation and the evaluation of speaker similarity in the EMIME speech-to-speech translation project. In SSW, pages 192-197, 2010. URL http://www. isca-speech.org/archive/ssw7/ssw7_192.html. 